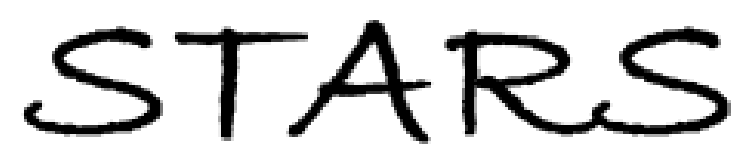

University of Central Florida

STARS

Faculty Bibliography 2000s

Faculty Bibliography

$1-1-2000$

\title{
Wide-angle, off-axis, see-through head-mounted display
}

Jannick P. Rolland

University of Central Florida

Find similar works at: https://stars.library.ucf.edu/facultybib2000

University of Central Florida Libraries http://library.ucf.edu

This Article is brought to you for free and open access by the Faculty Bibliography at STARS. It has been accepted for inclusion in Faculty Bibliography 2000s by an authorized administrator of STARS. For more information, please contactSTARS@ucf.edu.

\section{Recommended Citation}

Rolland, Jannick P., "Wide-angle, off-axis, see-through head-mounted display" (2000). Faculty Bibliography 2000s. 2774.

https://stars.library.ucf.edu/facultybib2000/2774

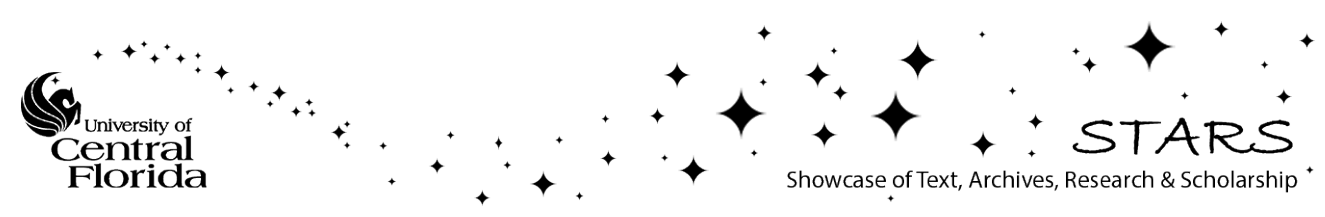




\section{Wide-angle, off-axis, see-through head-mounted display}

\author{
Jannick P. Rolland, MEMBER SPIE \\ University of Central Florida \\ School of Optics/CREOL \\ Orlando, Florida 32816
}

\begin{abstract}
A 60-deg-field-of-view optical see-through head-mounted display (HMD) using off-axis optics has been designed for 3-D medical imaging visualization. Two basic on-axis optical design concepts for seethough HMDs are reviewed first, to motivate the design of an off-axis optical form. An off-axis design is then presented. Because HMDs are typically designed from the pupil of the eye to the miniature display, it is common to assess final performance according to the display characteristics. Such analysis, however, does not provide information that is easily translated into task-based performance metric. Therefore, we present an analysis of the performance of the design from a usability viewpoint. For this analysis, the optical system is ray-traced from the display to the eye. Three key measures of performance-accommodation, astigmatism, and chromatic blur-are presented over the field of regard using customized graphical output. ๑ 2000 Society of Photo-Optical Instrumentation Engineers. [S0091-3286(00)00107-0]
\end{abstract}

Subject terms: medical imaging; head-mounted display.

Paper ENV-01 received Oct. 5, 1999; revised manuscript received Dec. 23, 1999; accepted for publication Jan. 6, 2000.

\section{Introduction}

With the rapid advance of three-dimensional (3-D) interactive computer graphics, binocular HMDs are finding applications in scientific as well as medical visualization. ${ }^{1,2}$ This is especially true of see-through devices. There are two types of see-through devices: optical and video. ${ }^{3-6}$ The design of an optical see-through HMD is presented in this paper. The requirements for the system, such as the field of view (FOV), the resolution, and the see-through capability, are driven in large part by the need to provide better interactive tools for the visualization of medical data, especially 3-D medical data.

An essential design criterion imposed by the dominant applications for binocular HMDs is the need to distinguish clearly virtual (e.g., computer-generated) objects superimposed on a bright background-a situation encountered, for example, in medical visualization, including surgical planning. To accomplish this, the brightness of the display must be high and the transmission of the optics from the image generator to the eyes must be controlled to ensure sufficient contrast of the virtual graphical objects when superimposed on a real-world scene. Moreover, the ability to display color information, while a less stringent criterion, is required for most applications in today's marketplace. These two criteria lead to the selection of a miniature color CRT as the display device. Finally, and importantly, given sufficient image contrast on the background, it is critical that the system does not suffer from ghost images. This latter criterion drives the design to an off-axis configuration, as further described in Sec. 2.

While wide FOV is not typically the most important requirement for a display to be used for medical visualization (30-deg FOV is typical), one of the requirements of this system was a 60-deg FOV for the first prototype. The use of the instrument in other virtual-reality applications, such as walkthrough, motivated the large FOV specification. $^{7}$ Other important constraints of the design were to minimize weight and to use a geometry that promotes comfort of the user when using the system for up to several hours.

An effective eye relief (eye clearance) of $23 \mathrm{~mm}$ is required in most production HMDs to allow for all types of eyeglasses. However, we selected an $18-\mathrm{mm}$ eye clearance (to accommodate only low-profile eyeglasses) for the prototype, so as to maximize FOV and peripheral vision. Furthermore, for optimum uniformity in brightness over the FOV, vignetting of less than $10 \%$ at the edge FOV was set as a design goal.

In the design of visual instruments, especially binocular instruments, the designer must allow for the natural ability of the wearers of the HMD to swivel their eyes in their sockets. As a result, even though the diameter of the pupil of the eye, which is the aperture stop for the optical system, is typically 3 to $5 \mathrm{~mm}$, the exit pupil size for the optical design was specified to be $10 \mathrm{~mm}$. This allows for an eye swivel of \pm 18 deg without causing vignetting in a 60 -deg FOV with a $3-\mathrm{mm}$ eye pupil diameter. ${ }^{8,9}$

Finally, we chose to optimize the design with respect to the optical aberrations that could not be compensated electronically or computationally. Optical system aberrations may cause either a decrease in image sharpness or a warping (distortion) of the shape of the objects being imaged. Warping of an object can be thought of as a change in magnification across the FOV. Two aberrations fall under this category: optical distortion and lateral color (change of transverse magnification with wavelength). Restricting the optimization to the minimization of optical aberrations that 
just cause a loss in image sharpness allows for the design of lighter systems.

Since the images are computationally generated, they can be prewarped in such a way as to cancel out the optical distortion generated through the optics. The final computergenerated images are perceived as sharp and undistorted. ${ }^{10-12}$

\section{On-Axis Design Concepts}

A critical component of any see-through optical system is the combiner, located in front of the eyes, that provides for the superposition of graphical and real-world information. We will review two basic design concepts, one based on a flat combiner and one based on a dual combiner. These are the two most commonly used for optical see-through HMDs. The size of the combiner will be shown to impose a constraint on the range of interocular distances allowed by the system. Typically, a span of interocular distances from 52 to $78 \mathrm{~mm}$ is desirable. This range, combined with the FOV, sets the size of the combiner at $50 \mathrm{~mm}$.

\subsection{Flat-Combiner Configuration}

A flat-combiner design consists of a miniature display, eyepiece optics, and a flat combiner. ${ }^{13}$ The main drawback of a flat-combiner system is the limitation on the maximum FOV to $40 \mathrm{deg}$, imposed by the minimum required eye relief and the large size of the combiner set by the range of interocular distances required. Furthermore, the size and consequently the weight of the eyepiece optics behind the combiner scale with the FOV. The main advantages of such a combiner are its simplicity and the high see-through transmission.

\subsection{Dual Combiner Configuration}

A dual combiner, made up from a spherical mirror combined with a half-silvered mirror, may be used to increase the FOV. ${ }^{14-16}$ The main advantage of such a system is that a FOV as high as 60 or 70 deg can be obtained, while at the same time all the components remain rotationally symmetric. The main drawbacks are the significant loss in seethrough transmission due to the multiple mirror reflections, and the existence of ghost images created by spurious reflections off the flat half-silvered mirror. These ghost images become unacceptable when the system is designed to be used in a bright-light environment such as medical imaging. This serious drawback led us to consider an off-axis design.

\section{Off-Axis Design}

An effective way to minimize ghost images is to use a single, tilted component combiner, as in the flat-combiner system, but with added optical power. This allows wider FOVs for the same effective eye relief. Adding power to a tilted combiner, however, introduces severe optical aberrations, such as coma, astigmatism, and asymmetric distortion, that must be compensated in the design of the relay optics.

Limiting the tilt of the combiner minimizes the off-axis aberrations. In the current design, the tilt of the combiner is 29.5 deg from the user's line of sight. Most important, and the key to developing a successful design, is to select a combiner of toroidal shape rather than spherical, to mini-

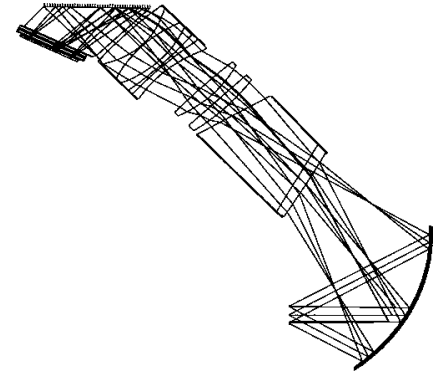

Fig. 1 Layout of the off-axis, 60-deg FOV HMD.

mize third-order astigmatism. Astigmatism is the dominant image-degrading aberration that is introduced by the offaxis configuration.

The two principal radii of curvature, $R_{x}$ and $R_{y}$, of the combiner that equalize the tangential $\left(T^{\prime}\right)$ and sagittal $\left(S^{\prime}\right)$ field curves (so as to minimize the effects of astigmatism), respectively, are given by the Coddington equations. ${ }^{17}$ For a mirror, the Coddington equations can be expressed as

$\left(\right.$ tangential) $\frac{1}{T^{\prime}}+\frac{1}{T}=\frac{2}{R_{y} \cos i}$

and

( sagittal) $\frac{1}{S^{\prime}}+\frac{1}{S}=\frac{2 \cos i}{R_{x}}$,

where $T, S$ and $T^{\prime}, S^{\prime}$ are the distances along the rays from the astigmatic focal surface to the focus for the object and image distances, respectively, and $i$ is the angle of the mirror with respect to the optical axis. To simplify the computation, we set the object at optical infinity and thus $1 / S$ and $1 / T$ to zero. If we solve Eq. (1) for $S^{\prime}$ equal to $T^{\prime}$, we obtain

$R_{x}=R_{y}(\cos i)^{2}$.

By setting $R_{x}=R \cos i, R_{y}=R / \cos i$, and assigning a value to $R$, starting-point values for $R_{x}$ and $R_{y}$ can be computed using Eq. (2). A value of $R$ equal to $80 \mathrm{~mm}$ was chosen as the starting point for the design developed here.

With the combiner selected, at least initially, the challenge is to design a relay lens, between the combiner and the miniature display, that compensates for the other offaxis aberrations while keeping the complexity for the lens to a minimum level and accommodating packaging requirements that are responsive to the needs of the user.

The relay lens we developed is shown Fig. 1. It contains two prisms to fold the optical system closely around the head of the wearer of the HMD. The folding geometry was established through an investigation in a virtual environment, where a 3-D model of a given design was positioned on virtual heads. The first prism (the prism closer to the combiner) also has the function of reducing the vignetting over the whole FOV. The design contains three nonspherical lenses: a cylindrical lens (located in the second lens after the first prism), and two parabolic lenses close to the second prism. The cylindrical lens, in conjunction with the ratio of the principal curvatures of the toroidal combiner predicted from Eq. (2), contributes to flattening the field. 
Table 1 Design specification.

\begin{tabular}{ll}
\hline \hline \multicolumn{1}{c}{ Parameter } & \multicolumn{1}{c}{ Specification } \\
\hline Configuration & Off-axis configuration \\
Object: miniature display & \\
a. Display type & Tektronix CRT \\
b. Active display area & Circular, 25.4-mm diameter \\
c. Resolution & $640 \times 480 ; 16-\mu \mathrm{m}$ pixel size \\
d. Color & Tektronix color shutter plate \\
e. Length & $101.6 \mathrm{~mm}$ (4 in.) \\
Eyepiece: & \\
a. Effective focal length & $23.7 \mathrm{~mm}$ \\
b. Exit pupil diameter & $10 \mathrm{~mm}$ \\
c. Object distance & $500 \mathrm{~mm}$ from eye entrance pupil \\
d. eye relief & $>17 \mathrm{~mm}(18 \mathrm{~mm}$ was achieved) \\
Other system parameters: & \\
a. Wavelength range & $597 \mathrm{to} 485 \mathrm{~nm}$ \\
b. Field of view & $60 \mathrm{deg}$ \\
c. Vignetting & $<10 \%$ over entire FOV \\
Image quality & \\
a. Distortion & \\
b. Image quality & $25-\mu \mathrm{m}$ rms spot size over FOV; \\
\hline \hline
\end{tabular}

The relay lens is a modified triplet lens where the types of glass have been chosen to minimize longitudinal and lateral chromatic aberrations. All elements, except the prisms, were made of either SK5 or SFL6 glass. The glass type for the folding prism closest to the display was chosen to have a high index to facilitate its insertion into the small clearance between the last lens and the miniature display image. Color was achieved by inserting a Tektronix color shutter plate close to the CRT. A small wedge prism between the 45-deg folding prism and the Tektronix plate was inserted to avoid total internal reflections at the color plate interface due to some of the rays impinging at high angles, of incidence.

\section{Optimization}

The optical specifications of the off-axis lens design are summarized in Table 1. An $f$ - $\tan \theta$ mapping for the lens requires a $22-\mathrm{mm}$ effective focal length, while an $f$ - $\theta$ mapping requires $24.3 \mathrm{~mm}$. The focal length was allowed to vary between those two values before choosing $23.7 \mathrm{~mm}$. The system was optimized with rays traced from the pupil to the miniature display, using CODE $\mathrm{V}^{\circledR}$ (software from Optical Research Associates, Pasadena, California) for a full unvignetted 10-mm pupil, a circular FOV of $60 \mathrm{deg}$, and an 18-mm effective eye relief. All the tilts and decenters were kept in the $Y Z$ plane. After initial optimization trials, where several elements were allowed to tilt and decenter, the tilts were reduced to those associated with the prism close to the combiner as seen in the optical layout.
Decenters were necessary on all the elements. Finally, the aspherics were limited to two and constrained to be parabolas to control prototype fabrication costs.

The design is symmetric about the $Y Z$ plane, requiring optimization across the full FOV in the $Y$ direction but only over half the FOV in the $X$ direction. Points along the object diagonal were also included.

\section{Design Performance}

As suggested by Shenker (1984), ${ }^{18}$ image quality for HMDs is most informative when assessed in visual space. At least three essential optical defects must be assessed: accommodation (defocusing across the FOV), astigmatism, and, for color displays, transverse chromatic smear. ${ }^{19} \mathrm{We}$ shall first present some conventional optical design analysis for the optimized system when ray-traced from the eye to the display, and complete the analysis by characterizing these three essential components of system performance from the display to the eye.

\subsection{Analysis with Respect to the Miniature Display}

The polychromatic diffraction MTFs for a centered 3-mm and the full $10-\mathrm{mm}$ pupil are presented for the tangential and sagittal rayfan plots across representative field angles: on axis, at vertical fields of \pm 21 and $\pm 30 \mathrm{deg}$, at horizontal fields of 21 and $30 \mathrm{deg}$, and at diagonal fields of $\pm 30 \mathrm{deg}$. Results for the $3-\mathrm{mm}$ and the $10-\mathrm{mm}$ pupil are shown in Fig. 2 and Fig. 3, respectively.

Astigmatism can be estimated from the difference in performance between the tangential and sagittal orientations. Residual astigmatism can also be quantified using the full-field astigmatic plot given in Fig. 4. This display uses methods developed by Coddington to characterize just the astigmatic component of image degradation. Here it is shown as a line whose length is proportional to the amount of astigmatism sampled over a grid of field points at the display. In Fig. 4, astigmatism is shown for a pupil of 3 $\mathrm{mm}$. We note that the residual astigmatism is well balanced over the whole FOV. The maximum line extent is $0.06 \mathrm{~mm}$ at the edge of the field. The average line extent is $0.03 \mathrm{~mm}$, or 1.9 pixels given a $16-\mu \mathrm{m}$ pixel size (see Table 1 ). Given a transverse magnification of 21.6 from the miniature display to the virtual image, one pixel subtends 2.4 arcmin at $0.5 \mathrm{~m}$. Therefore, a $0.03-\mathrm{mm}$ astigmatic line subtends a 4.6-arcmin visual angle. With respect to the medial focus, we thus predict a 2.3 -arcmin average resolution. An analysis, performed directly in visual space with respect to best focus, is also presented in Sec. 5.2.

The distortion of the lens is shown in Fig. 5. Distortion can be described as the sum of perspective (keystone) distortion that occurs uniquely in off-axis designs and the more conventional barrel distortion (plus some higher-order asymmetric components).

\subsection{Analysis in Visual Space}

The polychromatic diffraction MTFs, plotted against the spatial frequency in cycles per arcminute, were investigated for a centered 3-mm pupil and the tangential and sagittal ray fan plots across representative field angles: on axis; at vertical fields of $\pm 8.75 \mathrm{~mm}$ (i.e., $0.7 \mathrm{in}$ the field) and $\pm 12.5 \mathrm{~mm}$ (i.e., $1.0 \mathrm{in}$ the field); at horizontal fields of 8.75 

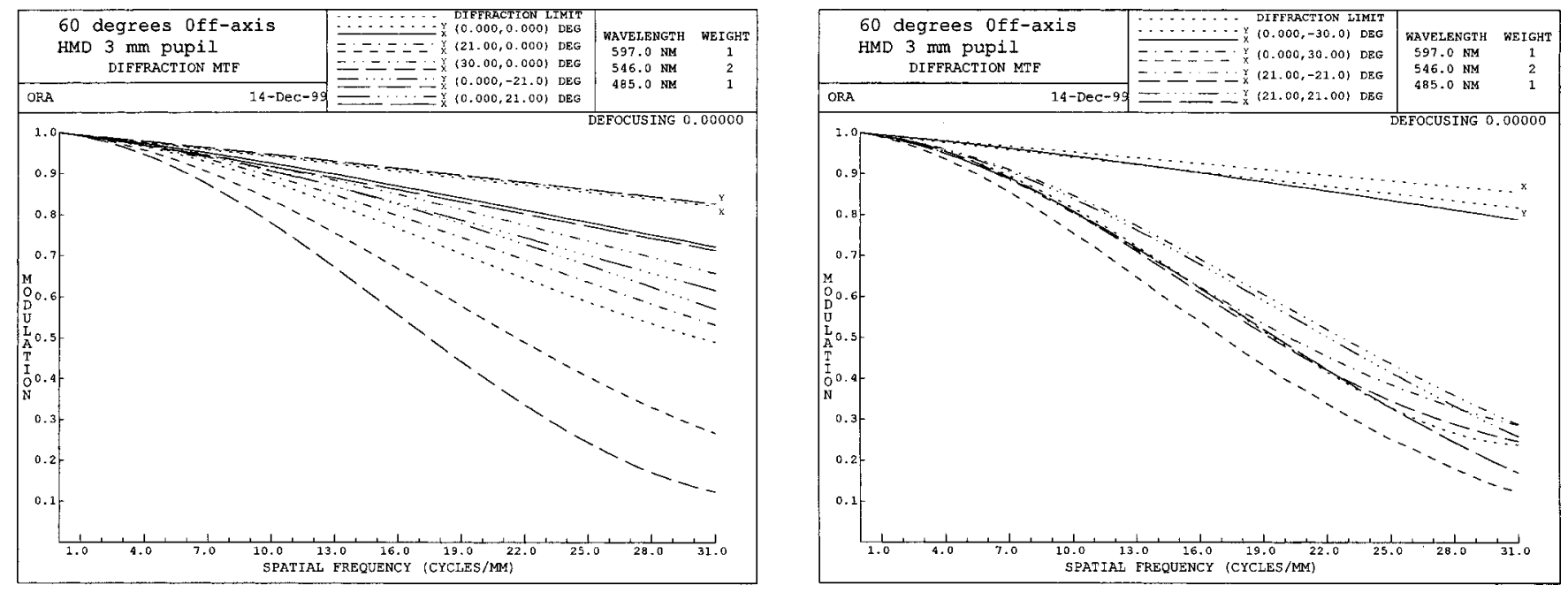

Fig. 2 Plots of the polychromatic MTF for a 3-mm pupil size and various points in the field of view. Plots are shown up to $31.25 \mathrm{lp} / \mathrm{mm}$, corresponding to a pixel size of $16 \mu \mathrm{m} ; 20 \mathrm{lp} / \mathrm{mm}$ corresponds to $25-\mu \mathrm{m}$ rms spot size, the desired performance.

$\mathrm{mm}$ (i.e., 0.7 in the field) and $12.5 \mathrm{~mm}$; and at diagonal fields of $\pm 12.5 \mathrm{~mm}$. The corresponding MTF plots are shown in Fig. 6.

Furthermore, a Zernike coefficient analysis conducted on an $11 \times 11$ grid over the display FOV clearly demonstrated that the system performance is dominated by only two aberrations, flatness of field (accommodation) and astigmatism. Other aberrations, such as coma and spherical aberration, were shown by the computations to be negligible, as were higher-order aberrations.

To further characterize the system from the perspective of a user, custom graphical outputs were developed in CODE $\mathrm{V}$ to display the accommodation and astigmatism over the field of regard in diopters. A 3-mm pupil size is assumed in all analyses pertaining to visual performance. The results are shown in Fig. 7. Here, as in Sec. 5.1, the ability to isolate the astigmatic component of the image degradation through the use of Coddington's differential ray-trace equations allows the performance analysis to highlight the two dominant measures of performance for visual systems. ${ }^{20}$

The accommodation, shown in Fig. 7(a), is relative to a focus that results in only positive accommodation values for the field of view, as negative accommodation is not physically allowed. As with the full-field astigmatic displays, the diameters of the circles are proportional to the amount of accommodation required when viewing a particular point on the display. The accommodation focal position is computed as the midpoint between the sagittal and tangential astigmatic curves that are based on Coddington's equations generalized to asymmetric optical systems. ${ }^{20}$

Requiring only positive accommodation over the full field led to a maximum of 1.3 diopters of refocus at the edge of the field. We note that 0.1 diopter of defocus around a virtual image at $0.5 \mathrm{~m}$ corresponds to 24 and 26 $\mathrm{mm}$ of defocus of the virtual image towards and away from
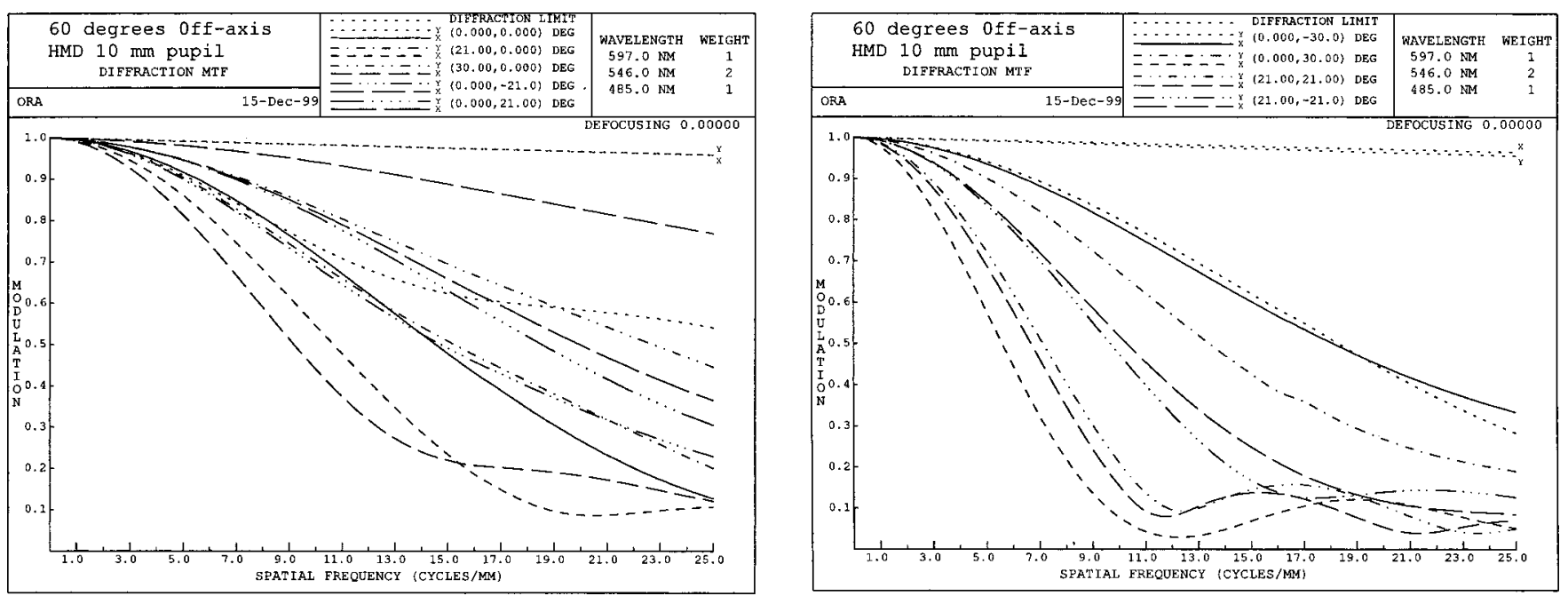

Fig. 3 Plots of the polychromatic MTF for a $10-\mathrm{mm}$ pupil size and various points in the FOV. Plots are shown up to $25 \mathrm{lp} / \mathrm{mm}$. 


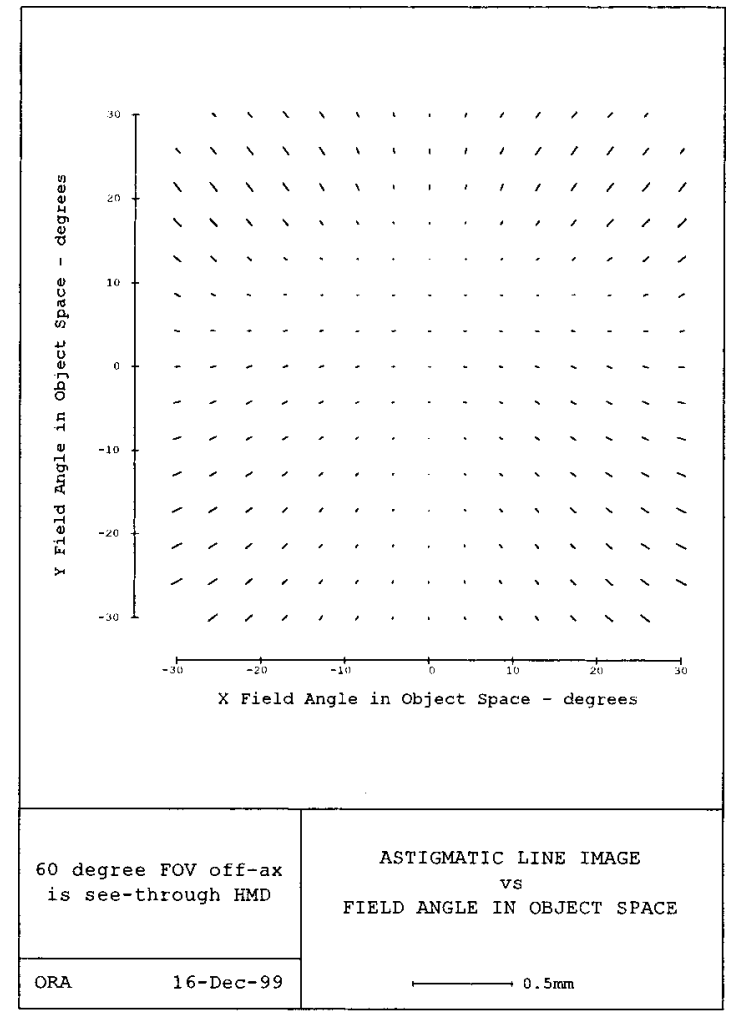

Fig. 4 Vector-field astigmatic plot. Within the 60-deg circular FOV, the maximum line length is $0.06 \mathrm{~mm}$, and the mean line length is $0.03 \mathrm{~mm}$. A pupil of $3 \mathrm{~mm}$ was selected.

the user, respectively. Thus, 0.1 diopter and a 3-mm pupil lead to 1 arcmin of loss in resolution. We thus predict 13 arcmin resolution at the worst point in the field, assuming the eye will accommodate at the best point. The figure shows the change from the center to the lower edge is about half this amount (i.e., 0.65 diopters), or 6.5-arcmin resolution. This performance is slightly lower than 5 arcmin required by the visual system at 30-deg visual angle off the

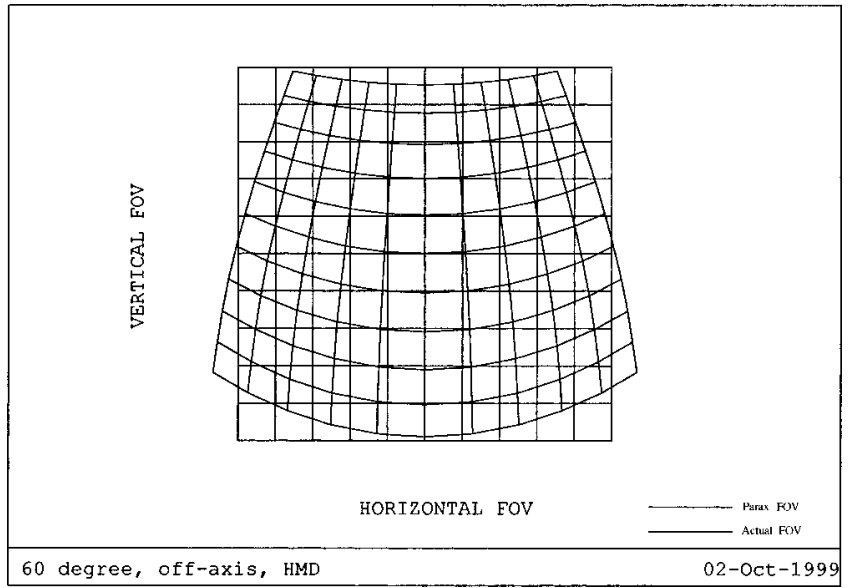

Fig. 5 Distortion of the off-axis, 60-deg FOV HMD.

eye gaze point. ${ }^{21}$ It is important to note, however, that most eyes can gaze at any point in the display and accommodate up to 2 diopters of focus shift, which is within the range of correction of this display.

Figure 7(b) is similar to the previous full-field astigmatic display, but in this case the magnitude of the focal shift between the sagittal and tangential astigmatic focal surfaces is shown, and it is given in diopters. Residual astigmatism across the FOV is less than or equal to 0.6 diopters. A loss in resolution of 3 arcminutes at the worst point in the FOV due to astigmatism will result for the current design, as the eyes can always choose best focus at the medial focus.

The performance in accommodation and astigmatism was also assessed as a function of eye movement within the exit pupil. We observed that the performance was nearly constant as a function of various decenters in the pupil, up to $5 \mathrm{~mm}$. The performance evaluated with a pupil decenter of $\pm 5 \mathrm{~mm}$ in $Y$ and $5 \mathrm{~mm}$ in $X$ is shown in Fig. 8 and Fig. 9 for accommodation and astigmatism, respectively.

To characterize the image smear due to residual color aberrations, Fig. 10 shows the difference in magnification
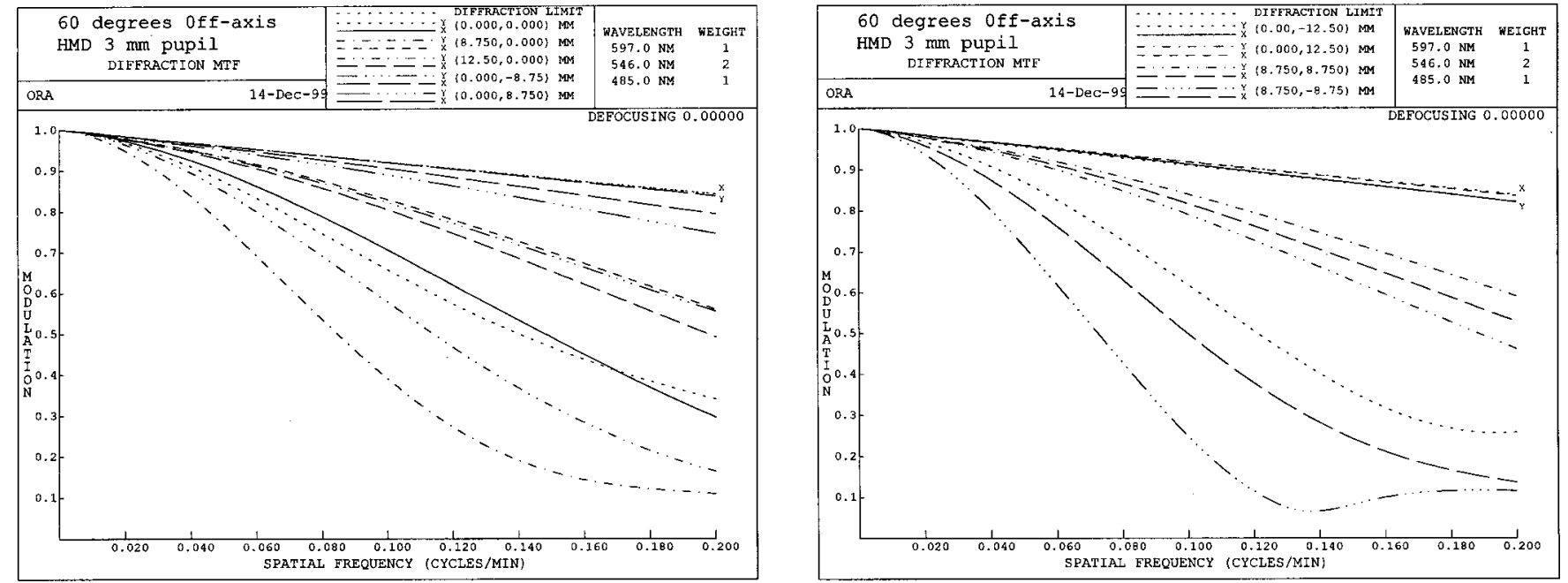

Fig. 6 Plots of the polychromatic MTF for a 3-mm pupil and various points in the field of view, plotted as a function of the spatial frequency (cycles/arcmin). Plots are shown up to 0.2 cycles/arcmin, corresponding to a 5 -arcmin resolution. 


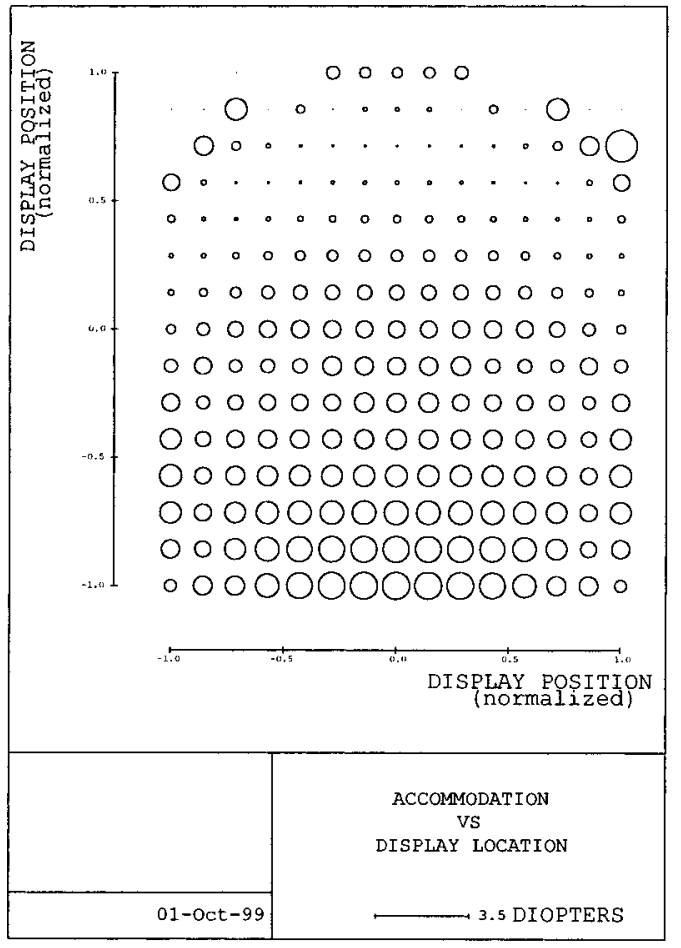

(a)

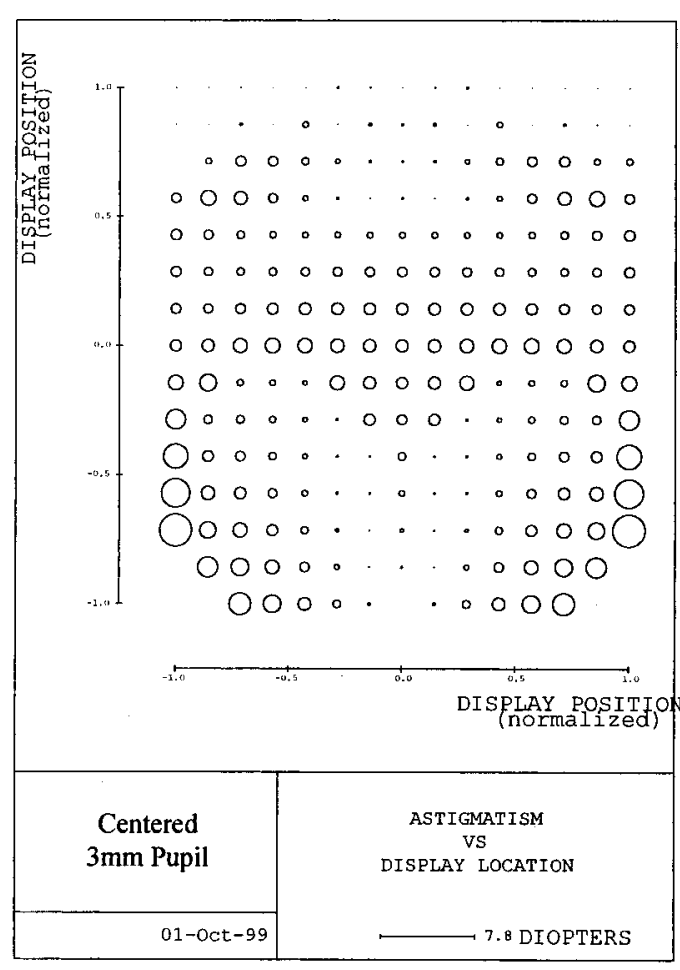

(b)

Fig. 7 Full-field (a) accommodation and (b) astigmatism plots for a centered 3-mm pupil. All values of accommodation are positive and are less than the 1.3 diopters obtained at the edge of the field. Values of astigmatism are less than or equal to 0.6 diopters within the 60 -deg circular FOV.

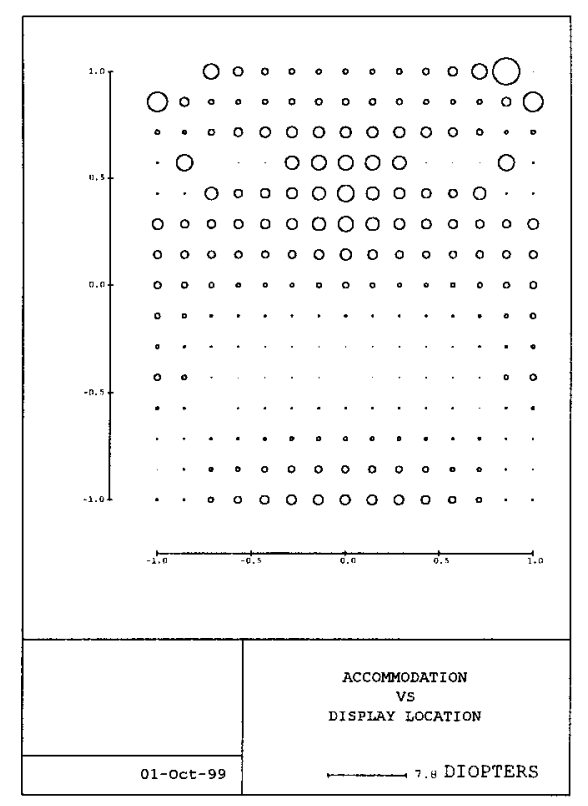

(a)

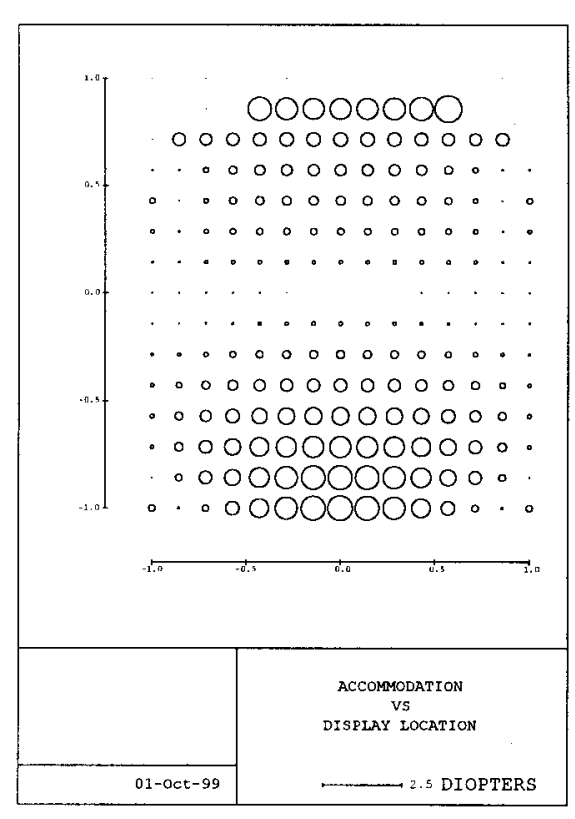

(b)

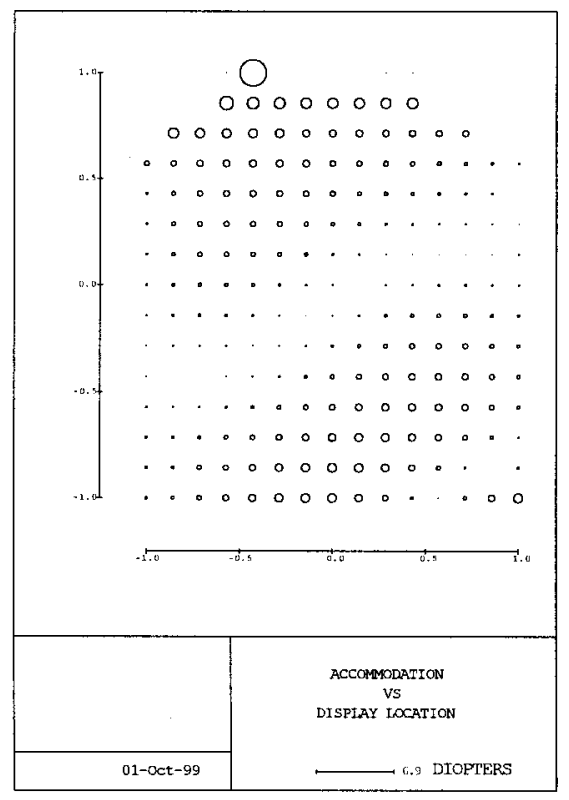

(c)

Fig. 8 Plots of accommodation over the FOV for (a) a +5 -mm $Y$-decentered pupil, (b) a $-5-\mathrm{mm}$ $Y$-decentered pupil, and (c) a +5 -mm $X$-decentered pupil. A 3-mm pupil size was selected. 


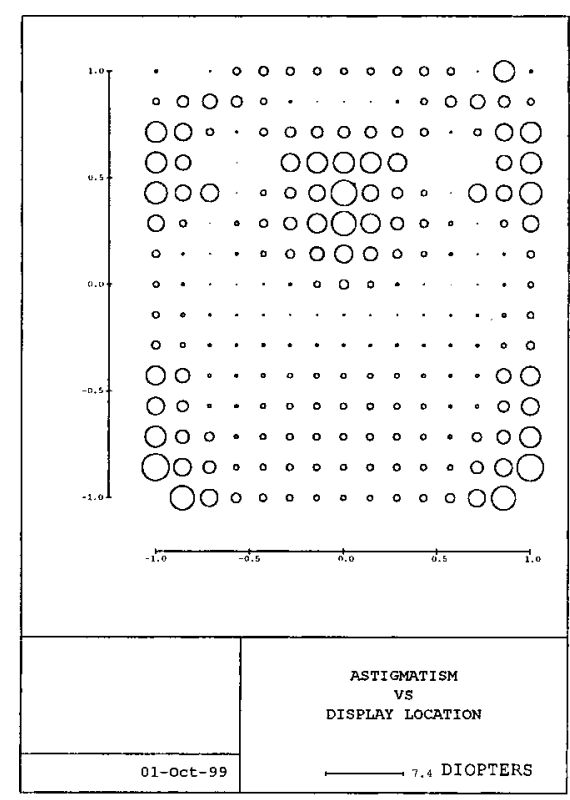

(a)

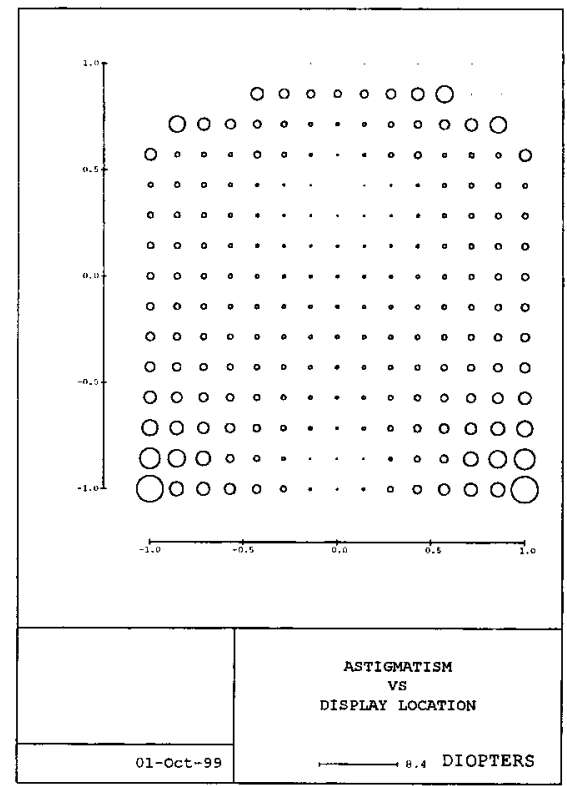

(b)

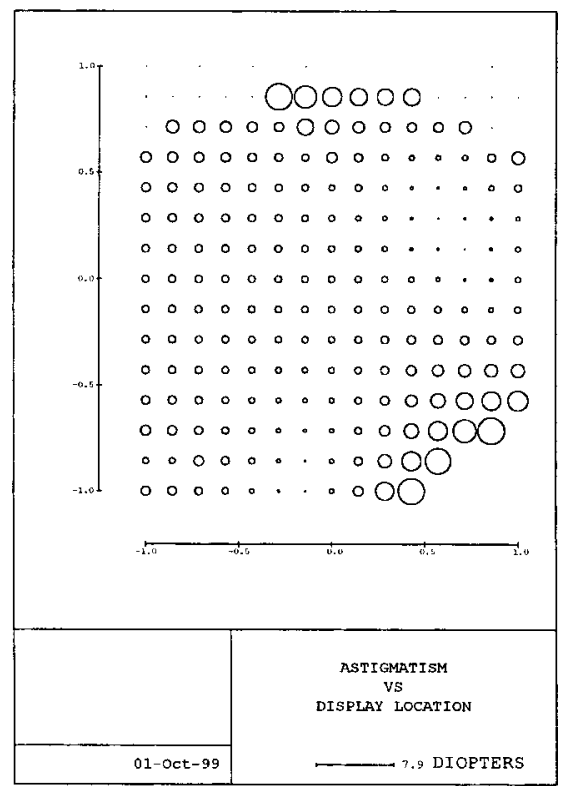

(c)

Fig. 9 Plots of astigmatism over the FOV is shown for (a) a $+5-\mathrm{mm} Y$-decentered pupil, (b) a $-5-\mathrm{mm}$ $Y$-decentered pupil, and (c) a +5 -mm $X$-decentered pupil. A 3-mm pupil size was selected.

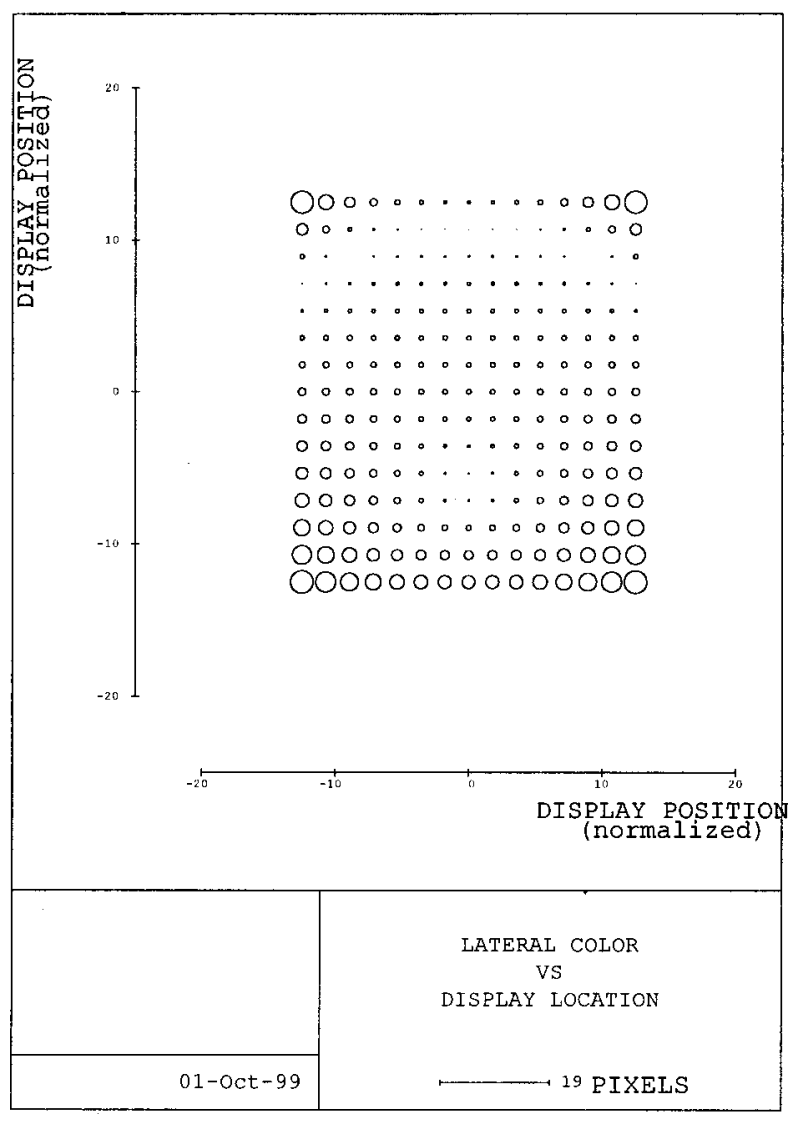

Fig. 10 Lateral color is shown over the full field. The maximum value at the edge of the field within the 60 -deg circular FOV is 1.6 pixels. between the two outer wavelengths over the field of view in arcminutes. As with Figs. 7-9, this display was developed using the spreadsheet feature in CODE V to accumulate the data of interest over the field of view, and the 2-D userdefined graphics features in CODE V to display them. The data consisted of the difference between the chief ray intercept in the displays at $597 \mathrm{~nm}$ and at $485 \mathrm{~nm}$, again over a 2-D grid of sample points in each display. This predicts the results of a three-color projection of a test grid of 1-pixel points on the display, a common performance test for HMDs.

\section{Conclusion}

The design of a 60-deg-FOV, color-corrected, off-axis optical system for head-mounted display was presented. The system was analyzed using both standard optical design software features and custom written CODE V graphics for analysis from the usability perspective. Within a circular 60-deg FOV, results show that for a 3-mm effective pupil size, astigmatism is fairly well balanced over the entire FOV and is estimated to cause a loss in resolution of 3 arcmin at the worst points in the FOV. The relative change in accommodation at the edge of the display with respect to the center of the display (assuming the eyes gaze at the center) is estimated to cause a loss in resolution of about 6.5 arcmin. However, as the eyes move, they can accommodate at various points in the field up to 2 diopters, which is within the range of correction of this display. Residual chromatic smear was estimated to be $<1$ pixel over $80 \%$ of the display format for this prototype design. 


\section{Acknowledgments}

I thank Henry Fuchs and Fred Brooks from the University of North Carolina at Chapel Hill (UNC-CH) for providing initial support to design the system. Special thanks go to Warren Robinett at UNC-CH for inspiring me to take on this project and his excellence in leadership that made the work possible. I thank Richard Buchroeder for stimulating discussions at various stages of the design and his warm encouragement through some of the challenging steps to completion. Finally, I would like to thank Martin Shenker of MSOD for suggesting that I assess the system visually, and Kevin Thompson of Optical Research Associates for his help in creating the custom CODE V graphics interface for analysis from the usability perspective, a key component of this paper. This design work was supported in part by DARPA under grants DAEA18-90-C-0044 and DABT63-93-C-0048, and the Office of Naval Research under grants N00014-94-1-0503 and N00014-97-10654. The visual analysis was supported by the National Institute of Health under grant 1-R29-LM06322-01A1.

\section{References}

1. D. L. Wright, J. P. Rolland, and A. R. Kancherla, "Using virtual reality to teach radiographic positioning," Radiol. Technol. 66(4), 167-172 (1995)

2. J. P. Rolland, 'Mounted displays,', Opt. Photonics News 9(11), 26-30 (1998).

3. R. A. Buchroeder, G. W. Seeley, and D. Vukobratovich, "Design of a catadioptric VCASS helmet-mounted display,' AFAMRL-TR-81133, Optical Sciences Center, Univ. of Arizona, under contract to U.S Air Force Armstrong Aerospace Medical Research Laboratory, Wright-Patterson Air Force Base, Dayton, OH (1981).

4. M. L. Thomas, W. P. Siegmund, S. E. Antos, and R. M. Robinson "Fiber optic development for use on the fiber optic helmet-mounted displays,' J. T. Carollo, Ed., Proc. SPIE 1116, 90-101 (1989).

5. F. Biocca and J. P. Rolland, "Virtual eyes can rearrange your body: adaptation to virtual eye location in see-thru head-mounted displays,' Presence: Teleoperators Virtual Environ. 7(3), 262-277 (1998).

6. J. P. Rolland and H. Fuchs, "A comparison of optical and video see-through head-mounted displays,' in Augmented Reality and Wearable Computers, W. Barfield and T. Caudell, Eds., Lawrence Erlbaum Associates, Mahwah, NJ (2000).

7. F. P. Brooks, "Walkthrough Project: final technical report to Nationa Science Foundation Computer and Information Science and Engineering,' Technical Report TR92-026, Univ. of North Carolina, Chapel Hill (1992)

8. D. F. Kocian, "Design considerations for virtual panoramic display (VPD) helmet systems," Armstrong Aerospace Medical Research Laboratory, Visual Display Systems Branch, Wright-Patterson Air Force Base, Dayton, OH (1988).
9. J. P. Rolland, A. Yoshida, L. Davis, and J. H. Reif, "High resolution inset head-mounted display," Appl. Opt. 37(19), 4183-4193 (1998).

10. W. Robinett and J. P. Rolland, "A computational model for the stereoscopic optics of a head-mounted display," Presence: Teleoperators Virtual Environ. 1(1), 45-62 (1992).

11. J. P. Rolland and T. Hopkins, "A method of computational correction for optical distortion in head-mounted displays,', Technical Report TR93-045, Univ. of North Carolina, Chapel Hill (1993).

12. B. Gharib, Y. Argotti, B. Peuchot, and J. P. Rolland, "Calibration of a see-through head-mounted display-application to augmented reality,' Technical Report TR99-005, Univ. of Central Florida (1999).

13. J. P. Rolland, D. Ariely, and W. Gibson, "Towards quantifying depth and size perception in virtual environments," Presence: Teleoperators Virtual Environ. 4(1), 24-49 (1995).

14. J. G. Droessler and D. J. Rotier, "Tilted cat helmet-mounted display,' Opt. Eng. 29(8), (1990).

15. M. L. Thomas and H. Geltmacher, "Combat simulator display development,' Inf. Disp. 9(4), 25 (1972).

16. J. P. Rolland, "Head-mounted displays for virtual environments: the optical interface," in OSA Proc. Int. Optical Design Conf. 22, G. W. Forbes, Ed., pp. 329-333 (1994).

17. H. Coddington, A Treatise on the Reflection and Refraction of Light, Part 1, Cambridge, UK (1829).

18. M. Shenker, "Image quality considerations for head-mounted displays,' ' in OSA Proc. Int. Optical Design Conf. 22, G. W. Forbes, Ed., pp. 334-338 (1994).

19. P. Mouroulis, T. G. Kim, and G. Zhao, "Transverse color tolerances for visual optical systems," Appl. Opt. 32, 7089-7094 (1993).

20. K. P. Thompson, "Aberrations fields in nonsymmetric optical systems," Ph.D. Dissertation, Univ. of Arizona (1980).

21. K. R. Boff, L. Kaufman, and J. P. Thomas, Handbook of Perception and Human Performance, Wiley, New York (1986).

Jannick Rolland is an assistant professor at the School of Optics and Center for Research and Education in Optics and Lasers (CREOL) at the University of Central Florida (UCF) in the United States. She also holds a joint appointment in the School of Electrical Engineering and Computer Science at UCF. She received an advanced optical engineering degree from the Ecole Superieure D'Optique in Orsay, France, in 1984, and her PhD from the University of Arizona, Optical Sciences Center, where her research focused on optical instrumentation and optical testing from 1984 to 1987 , and on medical imaging, and image quality assessment from 1987 to 1990. Dr. Rolland then joined the Department of Computer Science at the University of North Carolina (UNC) as a postdoctoral student to conduct research both in optical design for 3D visualization and medical imaging. She was appointed to the research faculty at UNC in 1992 and headed the Vision Research Group from 1992 to 1996. She joined CREOL in 1996 as a tenure earning faculty member. She holds two patents, wrote three book chapters and 22 peered review publications related to optical design, augmented reality, vision, and image quality assessment for medical imaging. She authored and co-authored over 50 other publications related to the same topics. 